\title{
Lung sensors in pulmonary diseases
}

\begin{abstract}
Sensory information in the lung is mainly generated by airway sensors carried by the vagus nerves, yielding multiple reflex responses essential for breathing control and lung defense. When these sensors are activated in lung disease, they produce clinical signs, including cough, bronchoconstriction and mucus secretion, and alter disease course by reflexes and neuroimmune interaction.
\end{abstract}

Keywords: lung disease, pulmonary receptors, vagus nerve
Volume 7 Issue 4 - 2020

\author{
Jerome Walker, Jerry Yu, ${ }^{1,2}$ \\ 'Robley RexVA Medical Center, USA \\ 2Pulmonary Division, Department of Medicine, University of \\ Louisville, USA
}

Correspondence: Jerry Yu, MD, PhD, Department of Medicine, Pulmonary Division, ACB-3, 530 South Jackson Street, University of Louisville, Louisville, KY 40292, Tel 502-852-5I46; Email j0yu000।@louisville.edu

Received: October 23, 2020 | Published: November 30, 2020
Abbreviations: SARs, slowly adapting receptors; RARs, rapidly adapting receptors; CFRs, C fiber receptors; DARs, deflationactivated receptors; HTARs, high threshold A $\delta$ receptors; NEBs, neuroepithelial bodies; COPD, chronic obstructive pulmonary disease; ARDS, acute respiratory distress syndrome; ELR, excitatory lung reflex; IEQ, inspiratory effort quotient; PI, inspiratory pressure; TXA2, thromboxane A2; NVS, NEBs-vagal system

\section{Introduction}

The lung and airway house an extensive network of mechanical and chemical sensors that regulate breathing and modulate defense reflexes and immune responses. Some are carried in sympathetic afferents, but the majority are in the vagal afferents. Until recently, the lung vagal sensors were categorized into three types: two mechanosensitive receptors [slowly adapting receptors (SARs) and rapidly adapting receptors (RARs)] innervated by myelinated fibers, and one chemosensitive $\mathrm{C}$ fiber receptors (CFRs) innervated by nonmyelinated fibers. With advancing research two additional myelinated sensor types have been added to the vagal sensory system: deflationactivated receptors (DARs) and high threshold A $\delta$ receptors (HTARs). The DARs are mechanosensitive and stimulated by lung deflation. They mediate pulmonary reflexes during acute pneumothorax. ${ }^{2}$ The HTARs are chemosensitive and innervated by myelinated A $\delta$ fibers, with an afferent conduction velocity of 4-15 m/s. Their afferent properties are more similar to CFRs than RARs and SARs. However, in contrast to CRFs, HTARs are not activated by phenyl diguanide. ${ }^{3}$

In addition to its chemosensors, the airway houses numerous neuroendocrine cells aggregated in neuroepithelial bodies (NEBs) that have been well characterized structurally. Although the NEBs are innervated by nerve fibers from different sources, including local ganglia, the spinal cord, and vagal nodose ganglia, their afferent activity has never been recorded or verified. They do, however, house numerous bioactive substances, neuropeptides, and mediators, including 5HT-an inflammatory mediator known to activate both CFRs and HTARs. Since NEBs connect with myelinated vagus nerve fibers, myelinated HTARs are likely candidates for their communication with CNS, that is, HTARs may transmit signals initiated or modulated by NEBs. ${ }^{4}$ This brief communication discusses the vagal airway sensors in lung disease with a focus on the chemosensors.

\section{Excitatory lung reflex}

Pulmonary diseases such as asthma, chronic obstructive pulmonary disease (COPD), acute respiratory distress syndrome (ARDS), pulmonary fibrosis, and pneumonia involve lesions in the lung parenchyma. These disorders emerge via multiple pathways operating within the lung, including activation of its chemosensitive HTARs and CFRs. The chemosensors are considered to be nociceptors since they are stimulated by hypertonic saline and various other noxious stimuli, including hydrogen peroxide, histamine, and bradykinin. To study chemosensory reflex effects in the lung parenchyma, we injected a minute amount of hypertonic saline into the lung $(0.05-0.1 \mathrm{ml}$, only $0.25 \%$ of the lung volume) to look at the respiratory responses in anesthetized, open chest, mechanically ventilated rabbits. An excitatory lung reflex (ELR) was observed comprised of tachypnea, hyperpnea, increased inspiratory time and inspiratory duty cycle, with decreasing expiratory time and expiratory muscle activity. ${ }^{5}$ Together these effects promote inspiratory muscle fatigue - quantifiable by a clinical measure referred to as the Inspiratory Effort Quotient (IEQ).

\section{IEQ $=$ PI/PImax, where PI = $(\mathrm{K}$ X VT/Cdyn $)$ X $($ TI/TTOT $)$}

Maximum inspiratory pressure (PImax) was held constant in these experiments. The inspiratory pressure (PI) terms are: K constant, VT tidal volume, Cdyn dynamic compliance, TI inspiratory time, and TTOT total breath time. Cdyn did not change during the measurement period, but phrenic nerve activity increased dramatically — associating with increased VT (neural VT, phrenic activity). This combination of inspiratory work stressors increases total ventilation and energy requirements to meet alveolar ventilation demands. Moreover, suppressing expiratory muscle activity puts further stress on the inspiratory muscles. Accordingly, activating sensors lying within the lung periphery associates with ensuing respiratory fatigue and risk of eventual respiratory failure. The major responsible sensors are believed to be HTARs. ${ }^{6}$ However, CFRs and DARs may also contribute to the reflex.

\section{Neuroimmune interaction}

Since chemosensors are activated by numerous agents (neurotransmitters, cytokines pro-inflammatory mediators), they may modulate disease processes in lung pathology and pathophysiology. Indeed, the chemosensors may act as biosensors to monitor proinflammatory cytokines propagated during inflammation, for example, in conditions such as infection and acute lung injury. In many diseases, inflammatory cytokines such as TNF- $\alpha$ and IL- $1 \beta$ are expressed by tissue and blood immune cells (including neutrophils, monocytes, NK cells and mast cells). Microinjection of these cytokines into the lung activates both HTARs and CFRs, supporting the hypothesis these chemosensors act as biosensors to detect the intensity of inflammatory 
response. ${ }^{7,8}$ There is further evidence the lung chemosensors detect inflammatory arachidonic acid (AA) metabolites. Pro-inflammatory thromboxane A2 (TXA2) mimetics stimulate the pulmonary CFRs and HTARs. Conversely, indomethacin, a cyclooxygenase inhibitor, and ketoconazole, a thromboxane synthetase inhibitor, suppress them. ${ }^{9}$ Reported elsewhere, the chemosensors are activated in a variety of conditions, such as intravenous injection of endotoxin, ${ }^{10}$ bradykinin, ${ }^{11}$ pulmonary air emboli, ${ }^{12}$ inhalation of wood smoke, ${ }^{13}$ and allergic asthma. ${ }^{14}$ The chemosensors also activate during acute lung injury induced by intravenous injection of Oleic Acid. ${ }^{15}$ It is interesting to note that intravenous injection of OA stimulates both HTARs and CFRs within seconds. These effects peaks within a minute and persist over a 90-minute observation period. Accordingly, different chemosensors may act in concert, generating dual information through both fast (HTARs) and slow (CFRs) tracks to modify the body's immune response. In the meantime, the activity of SARs and RARs also increases due to changes in lung mechanics, however, their responsiveness to mechanical stimulation decreases as ARDS develops. Clearly, mechanosensors and chemosensors are working together, but on different aspects, during diseased conditions.

Neuroimmune interaction influences the pathophysiological processes of lung diseases. Yet it is still an evolving area of research. For example, the NEBs-vagal system (NVS) may play an important role in the interaction at different levels (systemic, cellular, and molecular). We recently demonstrated the NVS can modulate the development of pulmonary fibrosis via neuro-immune interaction. More specifically, pulmonary fibrosis develops as the number and size of NEBs, pro-fibrogenic cytokines, and cells increase, as evidenced in mice intravenously injected with bleomycin. This pro-fibrotic process can be attenuated by interrupting the NVS. ${ }^{16}$

\section{Summary}

The lungs house various mechanosensors (RARs, SARs, and DARs) and chemosensors (CFRs and HTARs). Although these mechanosensors and chemosensors are quite different in their sensory behavior and reflex functions, and variably activate in lung diseases, understanding their potential clinical importance certainly will improve bedside management.

\section{Acknowledgement}

This study was supported in part by U.S. Department of Veteran Affairs Merit Review Award (PULM-024-17S).

\section{Conflicts of interest}

None.

\section{References}

1. Lee LY, Yu J. Sensory nerves in lung and airways. Compr Physiol. 2014;4(1):287-324.

2. Yu J. Deflation-activated sensors mediate pulmonary reflexes during acute pneumothorax. Gen Int Med Clin Innov. 2018;3(3):1-3.

3. Yu J. An overview of vagal airway receptors. Sheng Li Xue Bao. 2002;54(6):451-459.

4. Yu J, Lin SX, Zhang JW, et al. Pulmonary nociceptors are potentially connected with neuroepithelial bodies. Adv Exp Med Biol. 2006;580:301-306.

5. Yu J, Zhang JF, Fletcher EC. Stimulation of breathing by activation of pulmonary peripheral afferents in rabbits. $J$ Appl Physiol. 1985;85(4):1485-1492.

6. Yu J. In: Pandalai SG ed. Recent research developments in respiratory and critical care medicine. Research Signpost. 2002;55-68.

7. Li HF, Yu J. Airway chemosensitive receptors in vagus nerve perform neuro-immune interaction for lung-brain communication. Adv Exp Med Biol. 2009;648:421-426.

8. Yu J, Lin S, Zhang J, et al. Airway nociceptors activated by proinflammatory cytokines. Respir Physiol Neurobiol. 2007;156(2):116119.

9. Lin $\mathrm{S}$, Walker $\mathrm{J}, \mathrm{Xu} \mathrm{L}$, et al. Behaviours of pulmonary sensory receptors during development of acute lung injury in the rabbit. Exp Physiol. 2007;92(4):749-755.

10. Lai CJ, Ruan T, Kou YR. The involvement of hydroxyl radical and cyclooxygenase metabolites in the activation of lung vagal sensory receptors by circulatory endotoxin in rats. $J$ Appl Physiol. 2005;98(2):620-628.

11. Carr MJ, Kollarik M, Meeker SN, et al. A role for TRPV1 in bradykinininduced excitation of vagal airway afferent nerve terminals. J Pharmacol Exp Ther. 2003;304(3):1275-1279.

12. Chen HF, Lee BP, Kou YR. Mechanisms of stimulation of vagal pulmonary $\mathrm{C}$ fibers by pulmonary air embolism in dogs. J Appl Physiol (1985). 1997;82(3):765-771.

13. Lai CJ, Kou YR. Stimulation of vagal pulmonary C fibers by inhaled wood smoke in rats. J Appl Physiol (1985). 1998;84(1):30-36.

14. Zhang G, Lin RL, Wiggers M, et al. Altered expression of TRPV1 and sensitivity to capsaicin in pulmonary myelinated afferents following chronic airway inflammation in the rat. J Physiol. 2008;586(23):57715786.

15. Lin $\mathrm{S}, \mathrm{Li} \mathrm{H}, \mathrm{Xu} \mathrm{L}$, et al. Arachidonic acid products in airway nociceptor activation during acute lung injury. Exp Physiol. 2011;96(9):966-976.

16. Jain A, Yu J. Neuro-immune interactions during development of pulmonary fibrosis. Pulm Crit Care Med. 2017;2(3):1-3. 